\title{
Poles and zeros - examples of the behavioral approach applied to discrete linear repetitive processes
}

\author{
E. Rogers · P. M. Zaris · J. Wood - H. Pillai
}

Received: 14 April 2004 / Revised: 15 November 2005 /

Accepted: 17 November 2005 / Published online: April 2006

CSpringer Science+Business Media, LLC 2006

\begin{abstract}
In this paper the behavorial approach is applied to discrete linear repetitive processes, which are class of 2D systems of both systems theoretic and applications interest. The main results are on poles and zeros for these processes, which have exponential trajectory interpretations.
\end{abstract}

Keywords Repetitive processes - Behavioral approach $\cdot$ Poles and zeros

\section{Introduction}

Repetitive processes are a distinct class of 2D systems of both system theoretic and applications interest. The essential unique characteristic of such a process is a series of sweeps, termed passes, through a set of dynamics defined over a fixed finite duration known as the pass length. On each pass an output, termed the pass profile, is produced, which acts as a forcing function on, and hence contributes to, the dynamics of the next pass profile. This, in turn, leads to the unique control problem for these processes in that the output sequence of pass profiles generated can contain oscillations that increase in amplitude in the pass-to-pass direction.

To introduce a formal definition, let $\alpha<+\infty$ denote the pass length (assumed constant). Then in a repetitive process the pass profile $y_{k}(p), 0 \leq p \leq \alpha$, generated on pass $k$ acts as a forcing function on, and hence contributes to, the dynamics of the next pass profile $y_{k+1}(p), 0 \leq p \leq \alpha, k \geq 0$.

Physical examples of repetitive processes include long-wall coal cutting and metal rolling operations (see, for example, Benton, 2000 and the relevant references cited in this thesis).

\footnotetext{
E. Rogers $(\varangle) \cdot$ P. M. Zaris · J. Wood

School of Electronics and Computer Science, University of Southampton,

Southampton SO17 1BJ, UK

e-mail: etar@ecs.soton.ac.uk

H. Pillai

Department of Electrical Engineering, Indian Institute of Technology Bombay, Powai, Mumbai, 400 076, India
} 
Also in recent years applications have arisen where adopting a repetitive process setting for analysis has distinct advantages over alternatives. Examples of these so-called algorithmic applications include classes of iterative learning control (ILC) schemes (Owens, Amann, Rogers, \& French, 2000) and iterative algorithms for solving non-linear dynamic optimal control problems based on the maximum principle (Roberts, 2000). In the case of ILC for the linear dynamics case, the stability theory for so-called differential and discrete linear repetitive processes is the essential basis for a rigorous stability/convergence theory of one powerful class of such algorithms. For the non-linear optimal control algorithm, the repetitive process analysis has provided the essential basis for the development of highly reliable iterative solution algorithms.

In seeking a rigorous foundation on which to develop a control theory for these processes, it is natural to attempt to exploit structural links which exist between these processes and other classes of (2D) two-dimensional linear systems. The case of 2D discrete linear systems recursive in the positive quadrant $(i, j): i \geq 0, j \geq 0$ (where $i$ and $j$ denote the directions of information propagation) has been the subject of much research effort over the years using, in the main, the well known (Fornasini Marchesini, 1978) and (Roesser, 1975) state space models. It turns out, however, that large parts of established systems theory for 2D discrete linear systems described by these models either cannot be applied at all or only after appropriate modification. Hence there is a need to develop a systems theory for these processes for onward translation, where appropriate, into numerically reliable design algorithms.

This paper reports further development of two major aspects of such as systems theory. These are poles and zeros, which, unlike many others reported in the literature for 2D-dimensional linear systems, have a well defined interpretation in terms of exponential trajectories. These results are for the sub-class of so-called discrete linear repetitive processes, which arise in the modelling and control related analysis of a number of example application areas and also have well defined structural links with standard one - dimensional (1D) discrete linear systems. The pole and zero definitions and characterizations developed here are the natural generalizations of those for 1D discrete linear systems case.

\section{Background}

\subsection{Discrete linear repetitive processes}

The state space model of the sub-class of discrete linear repetitive processes considered here has the following form over $0 \leq p \leq \alpha-1, k \geq 0$,

$$
\begin{aligned}
x_{k+1}(p+1) & =A x_{k+1}(p)+B u_{k+1}(p)+B y_{k}(p), \\
y_{k+1}(p) & =C x_{k+1}(p)+D u_{k+1}(p)+D y_{k}(p) .
\end{aligned}
$$

Here on pass $k, x_{k}(p)$ is the $n \times 1$ state vector, $y_{k}(p)$ is the $m \times 1$ pass profile vector, and $u_{k}(p)$ is the $l \times 1$ vector of control inputs. To complete the process description it is necessary to specify the initial conditions, termed the boundary conditions here, i.e., the state initial vector on each pass and the initial pass profile. Here no loss of generality arises from assuming these to be zero.

Several equivalent sets of conditions for stability of these processes (in the strongest form), termed stability along the pass, are known but here we use the following. First define the along the pass and pass-to-pass shift operators as $\sigma_{1}$ and $\sigma_{2}$ applied, e.g., to $x_{k}(p)$ and $y_{k}(p)$ as $\sigma_{1} x_{k}(p):=x_{k}(p+1)$ and $\sigma_{2} y_{k}(p):=y_{k+1}(p)$, respectively. Consider also the 2D 
polynomial

$\mathcal{C}\left(\sigma_{1}, \sigma_{2}\right):=\operatorname{det}\left(\left[\begin{array}{cc}\sigma_{1} I_{n}-A & -B_{0} \\ -C & \sigma_{2} I_{m}-D_{0}\end{array}\right]\right)$.

Then it has been shown elsewhere (Rogers and Owens, 1992) that stability along the pass holds if, and only if,

$\mathcal{C}\left(\sigma_{1}, \sigma_{2}\right) \neq 0$ in $\mathcal{U}_{o c}\left(\sigma_{1}, \sigma_{2}\right):=\left\{\left(\sigma_{1}, \sigma_{2}\right):\left|\sigma_{1}\right| \geq 1,\left|\sigma_{2}\right| \geq 1\right\}$.

The 2D characteristic polynomial here reduces to its well known 1D linear systems counterpart if the previous pass terms are deleted from the repetitive process model (and the pass profile sub-script is dropped together with the finite pass length assumption). This suggests that the concept of a pole for the discrete linear repetitive processes considered here should be defined in terms of

$\mathcal{C}\left(\sigma_{1}, \sigma_{2}\right)=0$.

The task now is to make this formal where the best outcome would be a characterization of stability/instability in terms of trajectories defined by the pole locations, i.e. a generalization of the 1D linear systems case. In particular, we want to move away from a purely algebraic characterization to one which mimics the $1 \mathrm{D}$ linear systems case, e.g. poles generate exponential trajectories which characterize stability.

\subsection{Behavioral theory}

We view the system as a triple $(\mathcal{A}, q, \mathcal{B})$, where $\mathcal{A}$ the signal space is a vector space over the field $k=\mathbb{C}$ (or $\mathbb{R}$ ) or more generally a $\mathbb{K}[\sigma]$-module of $\mathrm{nD}$ mappings, where here $n=2$. The signal space $\mathcal{A}$ is taken as the discrete space $k^{\mathbb{N}}, q$ is the number of system variables and the behavior $\mathcal{B} \subseteq \mathcal{A}^{q}$ is the solution space of the finite set of $2 \mathrm{D}$ difference equations describing the process dynamics. For the repetitive processes considered here

$\mathcal{B}_{x, u, y}=\left\{\left(\begin{array}{l}x \\ u \\ y\end{array}\right) \in \mathcal{A}^{\bullet} \mid\left(\begin{array}{l}x \\ u \\ y\end{array}\right)\right.$ satisfy (1) $\}$.

Note that $\mathcal{A}^{\bullet}$ denotes the appropriate number of copies of the signal space $\mathcal{A}$.

For the polynomial ring $\mathbb{K}[\sigma]$, the ring action $\mathbb{K}[\sigma] \times \mathcal{A} \longrightarrow \mathcal{A}$ for discrete systems is defined as the shift operator $\sigma_{i}, i=1,2$. Using this notation we can write any repetitive process described by (1) in the form of a behavior, and similarly we can write any sub-process (such as when the output pass profile vector is zero - see below) in terms of sub-behaviors. For example, we can write (1) in a behavioral kernel representation, (see, e.g., Wood, Oberst, Rogers \& Owens, 2000),

$\mathcal{B}_{x, u, y}=\operatorname{Ker}_{\mathcal{A}}\left(\begin{array}{ccc}\sigma_{1} I_{n}-A & -\sigma_{2} B & -B_{0} \\ -C & -\sigma_{2} D & \sigma_{2} I_{m}-D_{0}\end{array}\right) \subseteq \mathcal{A}^{q}$,

where we use the suffix notation $\operatorname{Ker}_{\mathcal{A}}$ to denote the kernel of the ring action of the matrix. An important sub-behavior is the one containing all outputs that are zero, i.e., the sub-behavior

$\mathcal{B}_{x, u, 0}:=\left\{\left(\begin{array}{l}x \\ u \\ y\end{array}\right) \in \mathcal{B}_{x, u, y} \mid y=0\right\}$,

which we see is given by the kernel representation

$\mathcal{B}_{x, u, 0} \cong \operatorname{Ker}_{\mathcal{A}}\left(\begin{array}{cc}\sigma_{1} I_{n}-A & -\sigma_{2} B \\ -C & -\sigma_{2} D\end{array}\right)$ 
and also the zero input sub-behavior

$\mathcal{B}_{x, 0, y} \cong \operatorname{Ker}_{\mathcal{A}}\left(\begin{array}{cc}\sigma_{1} I_{n}-A & -B_{0} \\ -C & \sigma_{2} I_{m}-D_{0}\end{array}\right)$.

The first of these sub-behaviors will be very important when considering invariant zeros and the second when considering poles. For example, the invariant zeros in the 1D case are given by the set of points in $\mathbb{C}$ where the Rosenbrock system matrix loses rank, (see e.g., MacFarlane \& Karcanias, 1975). We will show in this paper that our definition of invariant zeros for discrete linear repetitive processes are the varieties in $\mathbb{C}^{2}$ such that a $2 \mathrm{D}$ polynomial matrix loses rank and hence our analysis will have to consider the rank loss points of such a matrix. We next outline some required preliminary results concerning behaviors (for a detailed treatment of behaviors see the cited references in, e.g. (Wood 2000) and the continually evolving literature).

Let $M$ be a finitely generated $\mathcal{R}$-module. The dual of $M$ with respect to $\mathcal{A}$, denoted $D(M)$, is defined by

$D(M):=\operatorname{Hom}_{\mathcal{R}}(M, \mathcal{A})$.

If $\psi: M \rightarrow N$ is a morphism of finitely generated $\mathcal{R}$-modules, then the dual map $D(\psi): D(N) \rightarrow D(M)$ is given by $\forall v \in D(N),(D(\psi))(v):=v \circ \psi$. Then results in Oberst (1990) tells us precisely what the module $M$ is but here the crucial fact used is that if $\mathcal{B}=\operatorname{Ker}_{\mathcal{A}} \mathrm{E}$ then $\mathcal{B}^{\perp}=\operatorname{Im}_{\mathcal{R}} E$ and then $M$ is the finitely generated module $\operatorname{Coker}_{\mathcal{R}} E=\operatorname{Coker}^{1, q} / \operatorname{Im}_{\mathcal{R}} E=\mathcal{R}^{1, q} / \mathcal{B}^{\perp}$.

The set of variables $\left\{w_{i} \in \Phi\right\}$ for some subset $\Phi$ of $\{1, \ldots, q\}$ is said to be a set of free variables if the mapping $\rho: \mathcal{A}^{q} \rightarrow \mathcal{A}^{\Phi}$, which projects a trajectory onto the components of $\phi$, is epic when restricted to $\mathcal{B}$. The maximum cardinality of such a set $\Phi$ is an invariant of the behavior and is denoted by $m(\mathcal{B})$, and is given by $m(\mathcal{B})=q-\operatorname{rank}(E)$ where $\mathcal{B}=\operatorname{Ker}_{\mathcal{A}} E$.

We define the annihilator of a behavior $\mathcal{B}$ as

ann $\mathcal{B}=\{s \in \mathcal{R} \mid s \widetilde{w}=0 \forall \widetilde{w} \in \mathcal{B}\}$.

From Wood et al. (2000) we have ann $\mathcal{B}=$ ann $M$. A behavior containing no free variables is an autonomous behavior and is precisely one which has a non-zero annihilator. In the discrete case, we define a controllable behavior (Wood, 2000) $\mathcal{B}$ with signal domain $T=\mathbb{N}^{n}$ to be controllable if there exits a number $\rho>0$ such that for any sets $T_{1}, T_{2} \subseteq T$ with $d\left(T_{1}, T_{2}\right)>\rho$, for any $\underline{b}_{1}, \underline{b}_{2} \in T$, and any two trajectories $w^{(1)}, w^{(2)} \in \mathcal{B}$, there exists $w \in \mathcal{B}$ such that

$w(\underline{t})= \begin{cases}w^{(1)}\left(\underline{t}-\underline{b}_{1}\right), & \text { if } \underline{t} \in T_{1} \text { and } \underline{t}-\underline{b}_{1} \in T, \\ w^{(2)}\left(\underline{t}-\underline{b}_{2}\right), & \text { if } \underline{t} \in T_{2} \text { and } \underline{t}-\underline{b}_{2} \in T,\end{cases}$

where we can take $\underline{b}_{1}=0$ without loss of generality. Controllability in this setting expresses the idea of being able to join with a system trajectory any two system trajectories defined on regions, which are sufficiently far apart. Characterizations for this property are well known in the literature and in the case of the discrete linear repetitive processes considered here further progress is possible (Rogers et al., 2002).

For a given behavior, we define the controllable part as the unique maximal controllable sub-behavior, and we denote this, the controllable part of $\mathcal{B}$, by $\mathcal{B}^{c}$. It is well known that for $\mathcal{B}=D(M)$,

$\mathcal{B}^{c}=D(M / t M)$ and $\mathcal{B} / \mathcal{B}^{c}=D(t M)$,

where $t M$ is the torsion submodule of $M$. 


\subsection{Characteristic and generalized characteristic varieties}

Linear systems with constant coefficients are entirely characterized by the exponential trajectories contained in their behavior. We now give the definition of such trajectories, again for the case of $n=2$, which is required here.

Definition 1 Let $w(t)=w\left(t_{1}, t_{2}\right) \in \mathcal{A}^{q}$. Then $w$ is said to be an exponential trajectory of frequency $\left(a_{1}, a_{2}\right) \in \mathbb{C}^{2}$ if it is of the form

$w(t)=v_{0} a_{1}^{t_{1}} a_{2}^{t_{2}}$,

where $v_{0} \in \mathcal{C}^{q}$. Also $w$ is said to be a polynomial exponential trajectory of pure frequency $\left(a_{1}, a_{2}\right)$ if it is of the form

$w(t)=p(t) a_{1}^{t_{1}} a_{2}^{t_{2}}$,

where $p(t)=p\left(t_{1}, t_{2}\right) \in \mathbb{C}\left[t_{1}, t_{2}\right]^{q}$. A polynomial exponential trajectory is any trajectory, which is a finite sum of polynomial trajectories of pure frequencies.

Let $J \subseteq \mathcal{R}$, be an ideal, where $k=\mathbb{R}$ or $\mathbb{C}$. Define the variety $V(J)$ as

$V(J):=\left\{a \in \mathbb{C}^{2} \mid p(a)=0 \forall p \in J\right\}$.

Note that $V(J)$ is defined as a subset of $\mathbb{C}^{2}$ even when $k=\mathbb{R}$.

Definition 2 (Wood et al., 2000) The characteristic variety of a behavior $\mathcal{B}=\operatorname{Ker}_{\mathcal{A}} R$ is the set $\mathcal{V}(\mathcal{B})$ of all points $\left(a_{1}, a_{2}\right) \in \mathbb{C}^{2}$ such that the following equivalent conditions hold:

1. $\left(a_{1}, a_{2}\right) \in \mathcal{V}(\operatorname{ann} \mathcal{B})$.

2. $R\left(a_{1}, a_{2}\right)$ has less than full column rank.

3. $\mathcal{B}$ contains a non-zero exponential trajectory of frequency $\left(a_{1}, a_{2}\right)$.

The points in $\mathcal{V}(\mathcal{B})$ are called the characteristic points of $\mathcal{B}$.

Note that if $\mathcal{B}$ contains a non-zero polynomial exponential trajectory of pure frequency $\left(a_{1}, a_{2}\right)$ then by repeated differentiation it also contains a non-zero exponential trajectory of the same frequency.

The next result provides a characterization of the characteristic variety of a factor behavior $\mathcal{B} / \mathcal{B}^{\prime}$.

Theorem 1 (Zaris, Wood \& Rogers, 2001). Let $\mathcal{B}^{\prime} \subseteq \mathcal{B}$ be behaviors and $\left(a_{1}, a_{2}\right) \in \mathbb{C}^{2}$. Then the following are equivalent;

1. $\left(a_{1}, a_{2}\right)$ is a characteristic point of $\mathcal{B} / \mathcal{B}^{\prime}$.

2. There exists a polynomial vector $x$ such that $x \tilde{w}^{\prime}=0$ for all $\tilde{w}^{\prime} \in \mathcal{B}^{\prime}$ but $x \tilde{w}$ equals a non-zero exponential trajectory of frequency $\left(a_{1}, a_{2}\right)$ for some $\tilde{w} \in \mathcal{B}$.

3. There exists a polynomial exponential trajectory of pure frequency $\left(a_{1}, a_{2}\right)$ in $\mathcal{B} \backslash \mathcal{B}^{\prime}$.

In order to define the zeros of a behavior, it is necessary to consider the rank loss points of the representation matrix of $\mathcal{B}$. By rank loss points we mean those values of $\left(a_{1}, a_{2}\right) \in \mathbb{C}^{2}$ such that the representation matrix of the behavior loses rank. Consider then the matrix $R \in \mathcal{R}^{g, q}$ of rank $b \leq q$, and let $I_{b}(R) \subset \mathcal{R}$ denote the ideal generated by the order $b$ minors of $R$. The rank loss points of $R$ are given by the elements of the variety of the ideal $I_{b}(R)$, that is by $V\left(I_{b}(R)\right)$. Moreover, the rank loss points of any kernel representation of $\mathcal{B}$ are precisely the generalized characteristic points of $\mathcal{B}$. Moreover, if $\mathcal{B}$ is autonomous (i.e., $M$ is a torsion module) then $\mathcal{V}(\mathcal{B})=V($ ann $M)$.

As with the characteristic variety, we have an interpretation of the generalized characteristic points in terms of exponential trajectories and rank loss points. 
Theorem 2 (Zaris et al., 2001). The following are equivalent for a behavior $\mathcal{B}=\operatorname{Ker}_{\mathcal{A}} R$ and $\left(a_{1}, a_{2}\right) \in \mathbb{C}^{2}$;

1. $\left(a_{1}, a_{2}\right) \in \mathcal{V}(\mathcal{B})$.

2. The rank of $R\left(a_{1}, a_{2}\right)$ is less than the rank of $R\left(z_{1}, z_{2}\right)$.

3. For any of up to $m(\mathcal{B})$ variables $\omega_{i}, \mathcal{B}$ contains a non-zero exponential trajectory of frequency $\left(a_{1}, a_{2}\right)$, which is zero in the specified components.

\section{Poles and zeros}

Consider first the case of poles where it is first appropriate to recall the 1D case. Then in this case point $a$ is a pole of the system (defined by the standard state space model with output $y(t)$, state vector $x(t)$, control input $u(t))$ if, when zero input $u(t)$ is fed to the system, there exists a non-zero initial condition $x(0)$ such that the resulting state trajectory has the form $x(t)=x(0) a^{t}$. Note that as $y(t)$ is determined linearly by $x(t)(u(t)$ being zero), $y(t)$ must also be of the form $y(0) a^{t}$.

In the case of the discrete linear repetitive processes considered here, the solutions of the defining equations can be considered as functions from $\mathbb{N}^{2}$ to $\mathbb{R}$, though for purposes of interpretation they are cut off in one dimension at the pass length $\alpha$.

The poles of the system are defined as the characteristic points of the zero-input behavior $\mathcal{B}_{x, 0, y}$, that is the set of all trajectories, which can arise when the input vanishes. The zero-input behavior is given (to within trivial isomorphism) by

$\left(\begin{array}{cc}\sigma_{1} I_{n}-A & -B_{0} \\ -C & \sigma_{2} I_{m}-D_{0}\end{array}\right)\left(\begin{array}{l}x \\ y\end{array}\right)=0$.

Applying Theorem/Definition 4.4 of Wood et al. (2000), we can define the poles as follows.

Definition 3 The poles of a linear repetitive process described by (1) are the component-wise non-zero points in 2D complex space where the matrix on the left-hand-side of (15) fails to have full rank; that is, they are given by the set

$\mathcal{V}\left(\mathcal{B}_{x, 0, y}\right)=\left\{\left(a_{1}, a_{2}\right) \in \mathbb{C}^{2} \mid \mathcal{C}\left(a_{1}, a_{2}\right)=0\right\}$,

where $\mathcal{C}\left(\sigma_{1}, \sigma_{2}\right)$ is defined by (2). The set $\mathcal{V}$ is called the pole variety of the process.

Since in this case the pole variety is given by the vanishing of a single $2 \mathrm{D}$ non-unit polynomial, it is guaranteed to be a $1 \mathrm{D}$ geometric set in 2D complex space, that is, a curve. In particular, the pole variety cannot be zero-dimensional (i.e., finite). This corresponds to the fact that proper principal ideals in the ring $\mathbb{C}\left[\sigma_{1}, \sigma_{2}\right]$ have codimension 1 . Note also that the pole variety is a complex variety, even though the entries of the matrices $A, B_{0}, C$, and $D_{0}$ are generally assumed to be real. This is essential in order to capture the full exponential-type dynamics of the process.

Poles can be interpreted in terms of exponential trajectories (Wood et al., 2000), which in the case of discrete repetitive processes have a clear physical interpretation. Assume therefore that $\left(a_{1}, a_{2}\right) \in \mathbb{C}^{2}$ is such that $\mathcal{C}\left(\sigma_{1}, \sigma_{2}\right)=0$, and write it in the form $a_{1}=r_{1} e^{l \theta_{1}}, a_{2}=r_{2} e^{l \theta_{2}}$ (with $r_{1}=0$ for $a_{1}=0$ and $r_{2}=0$ for $a_{2}=0$ ). The existence of such a 'zero' guarantees (Wood et al., 2000) (Theorem/Definition 4.4) the existence of an 'exponential trajectory' in the process having the form

$$
\begin{aligned}
& x_{k}^{\prime}(p)=x_{00}^{1} r_{1}^{p} r_{2}^{k} \cos \left(\theta_{1} p+\theta_{2} k\right)+x_{00}^{2} r_{1}^{p} r_{2}^{k} \sin \left(\theta_{1} p+\theta_{2} k\right) \\
& y_{k}^{\prime}(p)=y_{00}^{1} r_{1}^{p} r_{2}^{k} \cos \left(\theta_{1} p+\theta_{2} k\right)+y_{00}^{2} r_{1}^{p} r_{2}^{k} \sin \left(\theta_{1} p+\theta_{2} k\right) \\
& u_{k}^{\prime}(p)=0, \\
& \text { 国 Springer }
\end{aligned}
$$


where $x_{00}^{1}, x_{00}^{2} \in \mathbb{R}^{n}, y_{00}^{1}, y_{00}^{2} \in \mathbb{R}^{m}$, and at least one of these four is non-zero. This form of exponential trajectory has been characterized algebraically by Oberst (1995). Conversely, the existence of such a trajectory implies that $\mathcal{C}\left(r_{1} e^{l \theta_{1}}, r_{2} e^{l \theta_{2}}\right)=0$, i.e. the 'frequency' $\left(r_{1} e^{l \theta_{1}}, r_{2} e^{l \theta}\right)$ is a pole of the repetitive process.

In the case where $\left(a_{1}, a_{2}\right) \in \mathbb{R}^{2}$, it is straightforward to construct such trajectories from the 'zeroes'. Take $a_{1}$ and $a_{2}$ to be real numbers satisfying $\mathcal{C}\left(a_{1}, a_{2}\right)=0$. There must then exist a non-zero vector $\left(x_{00}, y_{00}\right) \in \mathbb{R}^{n+m}$ satisfying

$\left(\begin{array}{cc}a_{1} I_{n}-A & -B_{0} \\ -C & a_{2} I_{m}-D_{0}\end{array}\right)\left(\begin{array}{l}x_{00} \\ y_{00}\end{array}\right)=0$.

Now extend $\left(x_{00}, y_{00}\right)$ to a process trajectory by

$x_{k}^{\prime}(p)=x_{00} a_{1}^{p} a_{2}^{k}$,

$y_{k}^{\prime}(p)=y_{00} a_{1}^{p} a_{2}^{k}$,

$u_{k}^{\prime}(p)=0$.

A quick computation yields

$$
\begin{aligned}
x_{k}^{\prime}(p+1) & =a_{1} x_{00} a_{1}^{p} a_{2}^{k} \\
& =A x_{00} a_{1}^{p} a_{2}^{k}+B_{0} y_{00} a_{1}^{p} a_{2}^{k} \\
& =A x_{k}^{\prime}(p)+B u_{k+1}^{\prime}(p)+B_{0} y_{k}^{\prime}(p), \\
y_{k+1}^{\prime}(p) & =a_{2} y_{00} a_{1}^{p} a_{2}^{k} \\
& =D_{0} y_{00} a_{1}^{p} a_{2}^{k}+C x_{00} a_{1}^{p} a_{2}^{k} \\
& =D_{0} y_{k}^{\prime}(p)+C x_{k}^{\prime}(p) .
\end{aligned}
$$

proving that (21)-(23) indeed describes a solution of the process.

Returning to the general case (17)-(19), we see that if $\left|a_{2}\right|=r \geq 1$ then we have a non-zero exponential (or sinusoidal) state-output trajectory in the system, which tends towards infinity as the pass number increases (but may remain stable along any given pass). Conversely, if $\left|a_{2}\right|=r<1$ for all poles $\left(a_{1}, a_{2}\right)$, then no trajectory tends to infinity for a given value of $p$ as the pass number increases, but there may be trajectories tending to infinity along the pass. Thus we again run up against the distinction between asymptotic stability and stability along the pass. In order to avoid having trajectories of the form (17)-(19), which are unstable either along the pass or in the $k$-direction, we also need to avoid poles $\left(a_{1}, a_{2}\right)$ with $\left|a_{1}\right| \geq 1$. In other words, we need that the characteristic variety (16) of the zero-input behavior lies inside $\overline{\mathcal{P}}_{1}=\left\{\left(a_{1}, a_{2}\right) \in \mathbb{C}^{2}|| a_{1}|\leq 1| a \mid, \leq 1\right\}$.

The necessary and sufficient condition of (3) states that stability along the pass is equivalent to all poles being inside $\overline{\mathcal{P}}_{1}$ (recall the $1 \mathrm{D}$ case!). Equivalently, with zero input there should be no exponential/sinusoidal state-output trajectories, which tend to infinity either in the pass-to-pass direction or along the pass. (Valcher has obtained similar results for the more general setting of stability of 2D behaviors over the lattice $\mathbb{I}^{2}$; Valcher, 2000).

Note finally that poles of the discrete linear repetitive processes considered here can be decomposed into controllable and uncontrollable, observable and unobservable poles (again recall the 1D case!), as described in Wood et al. (2000). The only one of these sets which can be easily described is the set of unobservable poles, which give the (2D) frequencies, which can occur in the state when both input and output vanish. These are given by the rank-loss points of the matrix

$\left(\begin{array}{c}z_{1} I_{n}-A \\ -C\end{array}\right)$ 
and so indeed describe the defect of observability.

In the case of zeros for the discrete linear repetitive processes considered here, first recall the $1 \mathrm{D}$ case and, in particular, the system defined over some field $\mathbb{K}$, by the polynomial matrix description

$$
\begin{aligned}
A(z) x & =B(z) u \\
y & =C(z) x+D(z) u,
\end{aligned}
$$

where $z$ denotes the shift operator, $A, B, C, D$ are polynomial matrices over $\mathbb{K}[z]$ and $x, u, y$ are state variables, input and output variables, respectively. Then the invariant zeros of a 1D discrete linear system are the rank loss points of its Rosenbrock system matrix

$$
P(z)=\left[\begin{array}{cc}
A(z) & -B(z) \\
C(z) & D(z)
\end{array}\right]
$$

and it is easy to see that this corresponds to the behavior $\mathcal{B}_{x, u, 0}$ and therefore the invariant zeros are given by the generalized characteristic points of the behavior $\mathcal{B}_{x, u, 0}$, i.e.,

$\{$ invariant zeros $\}=\mathcal{V}\left(\mathcal{B}_{x, u, 0}\right)$,

where we term $\mathcal{V}\left(\mathcal{B}_{x, u, 0}\right)$, the invariant zero variety.

We can easily generalize this to $\mathrm{nD}$ systems and, in particular, for the behavior $\mathcal{B}_{x, u, y}$ of (7) we can define the invariant zero points to be the elements of the variety $\mathcal{V}\left(\mathcal{B}_{x, u, 0}\right)$. As expected we can extend this concept very easily to define controllable and uncontrollable invariant zeros etc., to develop a zero structure - the structure $\mathcal{B}_{x, u, y}$ itself provides a map for this. The following pair of exact commutative diagrams demonstrate the structure of the behavior $\mathcal{B}_{x, u, y}$,
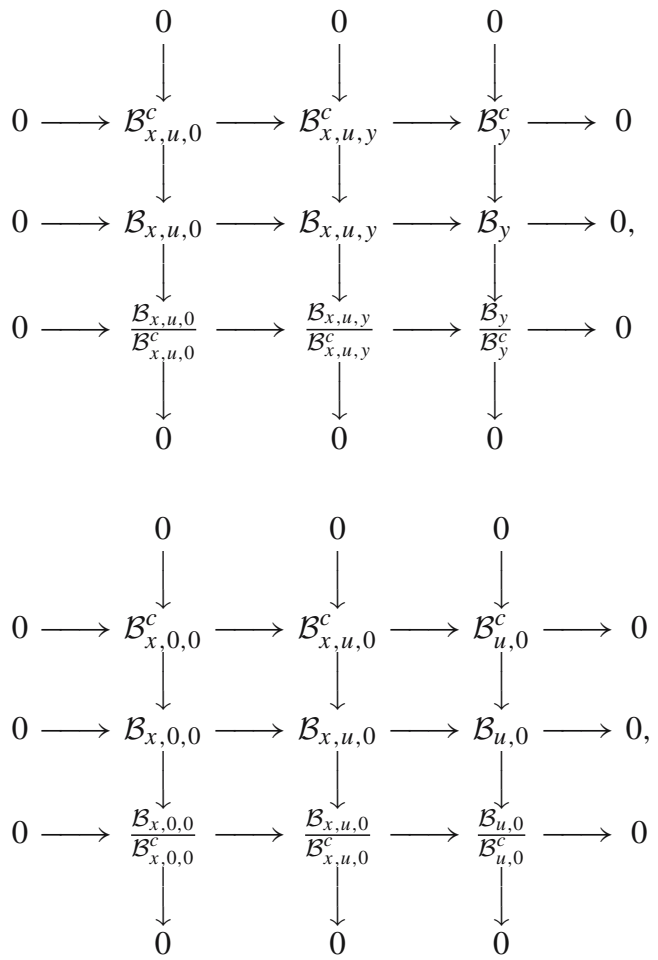
where

$$
\begin{aligned}
& \mathcal{B}_{y}:=\left\{y \in \mathcal{A}^{m} \mid \exists\left(\begin{array}{l}
x \\
u
\end{array}\right) \in \mathcal{A}^{n+l} ;\left(\begin{array}{l}
x \\
u \\
y
\end{array}\right) \in \mathcal{B}_{x, u, y}\right\}, \\
& \mathcal{B}_{x, 0,0}:=\left\{\left(\begin{array}{l}
x \\
u \\
y
\end{array}\right) \in \mathcal{B}_{x, u, y} \mid u=y=0\right\}, \\
& \mathcal{B}_{u, 0}:=\left\{\left(\begin{array}{l}
u \\
0
\end{array}\right) \in \mathcal{A}^{m+p} \mid \exists x \in \mathcal{A}^{l} ;\left(\begin{array}{l}
x \\
u \\
0
\end{array}\right) \in \mathcal{B}_{x, u, y}\right\} .
\end{aligned}
$$

We make the following definitions:

Definition 4 For the behavior $\mathcal{B}_{x, u, y}$ we have the following:

(i) The invariant [invariant controllable] zero variety is defined to be $\mathcal{V}\left(\mathcal{B}_{x, u, 0}\right)\left[\mathcal{V}\left(\mathcal{B}_{x, u, 0}^{c}\right)\right]$ and the invariant [invariant controllable] zero points as the elements of $\mathcal{V}\left(\mathcal{B}_{x, u, 0}\right)$ $\left[\mathcal{V}\left(\mathcal{B}_{x, u, 0}^{c}\right)\right]$.

(ii) The invariant uncontrollable zero variety is defined to be $\mathcal{V}\left(\mathcal{B}_{x, u, 0} / \mathcal{B}_{x, u, 0}^{c}\right)$ and the invariant uncontrollable zero points as the elements of $\mathcal{V}\left(\mathcal{B}_{x, u, 0} / \mathcal{B}_{x, u, 0}^{c}\right)$.

(iii) The observable [observable controllable] zero variety is defined to be $\mathcal{V}\left(\mathcal{B}_{u, 0}\right)$ $\left[\mathcal{V}\left(\mathcal{B}_{u, 0}^{c}\right)\right]$ and the observable [observable controllable] zero points as the elements of $\mathcal{V}\left(\mathcal{B}_{u, 0}\right)\left[\mathcal{V}\left(\mathcal{B}_{u, 0}^{c}\right)\right]$.

(iv) The observable uncontrollable zero variety is defined to be $\mathcal{V}\left(\mathcal{B}_{u, 0} / \mathcal{B}_{u, 0}^{c}\right)$ and the observable uncontrollable zero points as the elements of $\mathcal{V}\left(\mathcal{B}_{u, 0} / \mathcal{B}_{u, 0}^{c}\right)$.

From Wood et al. (2000) the uncontrollable pole points are defined to be the elements of the variety $\mathcal{V}\left(\mathcal{B}_{x, u, y} / \mathcal{B}_{x, u, y}^{c}\right)$.

Now we have the following result - for a proof (see Zaris, 2000).

Theorem 3 For the behavior $\mathcal{B}_{x, u, y}$ we have

(i) The invariant zero points are precisely the union of the invariant controllable and invariant uncontrollable zero points. That is

$\mathcal{V}\left(\mathcal{B}_{x, u, 0}\right)=\mathcal{V}\left(\mathcal{B}_{x, u, 0}^{c}\right) \cup \mathcal{V}\left(\mathcal{B}_{x, u, 0} / \mathcal{B}_{x, u, 0}^{c}\right)$

(ii) The observable zero points are precisely the union of the observable controllable and observable uncontrollable zero points. That is

$\mathcal{V}\left(\mathcal{B}_{u, 0}\right)=\mathcal{V}\left(\mathcal{B}_{u, 0}^{c}\right) \cup \mathcal{V}\left(\mathcal{B}_{u, 0} / \mathcal{B}_{u, 0}^{c}\right)$.

(iii) The invariant uncontrollable zero points are precisely the union of the unobservable uncontrollable and observable uncontrollable zero points. That is,

$\mathcal{V}\left(\mathcal{B}_{x, u, 0} / \mathcal{B}_{x, u, 0}^{c}\right)=\mathcal{V}\left(\mathcal{B}_{x, 0,0} / \mathcal{B}_{x, 0,0}^{c}\right) \cup \mathcal{V}\left(\mathcal{B}_{u, 0} / \mathcal{B}_{u, 0}^{c}\right)$.

(iv) The invariant uncontrollable zero points are contained in the uncontrollable pole points. In general we have

$\mathcal{V}\left(\mathcal{B}_{x, u, 0} / \mathcal{B}_{x, u, 0}^{c}\right)=\mathcal{V}\left(\mathcal{B}_{x, u, y} / \mathcal{B}_{x, u, 0}^{c}\right) \cup \mathcal{V}\left(\mathcal{B}_{y} / \mathcal{B}_{y}^{c}\right)$ 
(v) The invariant [controllable invariant] zero points are contained in the union of the unobservable [controllable unobservable] and observable [controllable observable] zeros, i.e.

$$
\begin{aligned}
& \mathcal{V}\left(\mathcal{B}_{x, u, 0}\right) \subset \mathcal{V}\left(\mathcal{B}_{x, 0,0}\right) \cup \mathcal{V}\left(\mathcal{B}_{u, 0}\right), \\
& \mathcal{V}\left(\mathcal{B}_{x, u, 0}^{c}\right) \subset \mathcal{V}\left(\mathcal{B}_{x, 0,0}^{c}\right) \cup \mathcal{V}\left(\mathcal{B}_{u, 0}^{c}\right) .
\end{aligned}
$$

In fact we can now show that the transmission zeros are not only contained in the invariant zeros but in the controllable invariant zeros - a subset of the invariant zeros. We need the following result from Zaris (2000).

Lemma 1 For any $1 D$ differential/difference behavior $\mathcal{B}=D(M)$, and any submodule $L \subset M$, we have for $\mathcal{B}^{\prime}=D(L)$, that $\mathcal{V}\left(\mathcal{B}^{\prime}\right) \subset \mathcal{V}(\mathcal{B})$.

As we have already noted the invariant zeros in the classical framework correspond to the invariant zeros in the behavioral framework. Similarly the transmission zeros correspond to the observable controllable zeros. Therefore applying Lemma 1 to the exact commutative diagram (24), we have the following results for the 1D case:

(i) The observable zero variety is contained in the invariant zero variety. That is

$$
\mathcal{V}\left(\mathcal{B}_{u, 0}\right) \subset \mathcal{V}\left(\mathcal{B}_{x, u, 0}\right)
$$

(ii) The observable controllable zero variety is contained in the invariant controllable zero variety. That is

$$
\mathcal{V}\left(\mathcal{B}_{u, 0}^{c}\right) \subset \mathcal{V}\left(\mathcal{B}_{x, u, 0}^{c}\right)
$$

From (ii), we therefore see in the 1D case that the transmission zeros (observable controllable zeros) are certainly contained in the invariant zeros (since the invariant zeros are the union of the invariant controllable and invariant uncontrollable zeros). More precisely, we see that they are in fact contained in the invariant controllable zeros.

We have the following physical characterization of invariant zeros in terms of exponential and polynomial exponential trajectories.

Theorem 4 (Zaris, 2000) Let $\mathcal{B}_{x, u, 0}^{c} \subset \mathcal{B}_{x, u, 0}$ where always $m^{\prime}=m\left(\mathcal{B}_{x, u, 0}^{c}\right)=m\left(\mathcal{B}_{x, u, 0}\right)$. Then we have the following;

(i) The point $\zeta \in \mathbb{C}^{n}$ is an invariant [resp. controllable] zero point of $\mathcal{B}$ if, and only if, for any choice of up to $m^{\prime}$ free (input) variables, there exists a non-zero exponential trajectory of frequency $\zeta$ contained in $\mathcal{B}_{x, u, 0}\left[\right.$ resp. $\left.\mathcal{B}_{x, u, 0}^{c}\right]$ with given choice of variables set to zero.

(ii) The point $\zeta \in \mathbb{C}^{n}$ is an invariant uncontrollable zero point of $\mathcal{B}$ if and only if there exists a non-zero polynomial exponential trajectory of frequency $\zeta$ contained in $\mathcal{B}_{x, u, 0}$ but not in $\mathcal{B}_{x, u, 0}^{c}$.

We now consider the case when the behavior $\mathcal{B}_{x, u, y}$ is a $2 \mathrm{D}$ behavior associated with discrete linear repetitive processes of the form considered here. We shall refer to the unobservable pole points as output decoupling zeros, and the unobservable uncontrollable pole points as the input-output decoupling zeros, and the observable controllable zero points as the transmission zeros. We have;

Theorem 5 Let $\mathcal{B}_{x, u, y}$ be an $2 D$ behavior as described above for the discrete linear repetitive processes considered here. Then we have the following. 
(i) The uncontrollable observable zeros and input output decoupling zeros are contained in the invariant zeros. That is

$\{$ i.o.d.z $\} \cup\{$ unc. obs. zeros $\} \subset\{$ invariant zeros $\}$.

(ii) If $\mathcal{B}_{x, u, y}$ is such that the outputs y are free, then the input decoupling zeros are contained in the invariant zeros. That is

$\{$ input dec. zeros $\} \subset\{$ invariant zeros $\}$.

(iii) If $\mathcal{B}_{x, u, 0}$ is autonomous then the transmission zeros and output decoupling zeros are contained in the invariant zeros. That is

\{output dec. zeros $\} \subset\{$ invariant zeros $\}$

$\{$ transmission zeros $\} \subset\{$ invariant zeros $\}$

At this stage return to the kernel representation of (5). Then given this representation, we can find kernel representation of the controllable part by first supposing that

$\mathcal{B}_{x, u, y}^{c}=\operatorname{Ker}_{\mathcal{A}}\left(R_{x}^{c} R_{u}^{c} R_{y}^{c}\right) \subseteq \mathcal{A}^{q}$.

Then we have

$\mathcal{B}_{x, 0,0}=\operatorname{Ker}_{\mathcal{A}}\left(\begin{array}{l}A \\ C\end{array}\right)$,

$\mathcal{B}_{x, 0,0}^{c}=\operatorname{Ker}_{\mathcal{A}}\left(R_{x}^{c}\right)$.

Similarly, by eliminating the latent variables $x$ we get

$\mathcal{B}_{u, y}=\operatorname{Ker}_{\mathcal{A}}\left(R_{u}^{m} R_{y}^{m}\right)$,

$\mathcal{B}_{u, y}^{c}=\operatorname{Ker}_{\mathcal{A}}\left(R_{u}^{m c} R_{y}^{m c}\right)$,

$\mathcal{B}_{0, y}^{c}=\operatorname{Ker}_{\mathcal{A}}\left(R_{y}^{m c}\right)$,

$\mathcal{B}_{u, 0}^{c}=\operatorname{Ker}_{\mathcal{A}}\left(R_{u}^{m c}\right)$.

Now, the output decoupling zeros are the rank loss points of $\left({ }_{C}^{A}\right)$, the transmission zeros are the rank loss points of $R_{u}^{m c}$ and the invariant zeros are the rank loss points of $\left(\begin{array}{cc}A & -B \\ C & D\end{array}\right)$. For the case of input decoupling zeros and input-output decoupling zeros, first find a kernel representation of the corresponding behaviors. Then the rank loss points of the kernel representations give the corresponding zeros.

\section{Conclusions}

The behavorial approach to systems theory has been used in the context of developing a polezero theory for discrete linear repetitive processes. The emphasis has been on developing definitions which have characterizations in terms of the trajectories produced by a given process with the eventual aim of using these to aid in the specification and design of control laws.

\section{References}

Benton, S.E. (2002). Analysis and control of linear repetitive processes. PhD Thesis, University of Southampton, UK. 
Fornasini, E., \& Marchesini, G. (1978). Doubly indexed dynamical systems: state models and structural properties. Mathematical Systems Theory, 12, 59-72.

MacFarlane, A. G. J.\& Karcanias, N. (1975). Poles and zeros of linear multivariable systems: a survey of the algebraic, geometric and complex-variable theory. International Journal of Control 24, 33-74.

Oberst, U. (1990). Multidimensional constant linear systems. Acta Applicande Mathematicae, 20, 1-175.

Oberst, O. (1995). Variations on the fundamental principle for linear systems of partial differential and difference equations with constant coefficients. Applicable Algebra in Engineering, Communication and Computing, 6(4-5), 211-243.

Owens, D. H., Amann, N., Rogers, E., \& French, M. (2000). Analysis of linear iterative learning control schemes - a 2D systems/repetitive processes approach. Multidimensional Systems and Signal Processing, $11(1-2), 125-177$.

Roberts, P. D. (2002). Numerical investigation of a stability theorem arising from 2-dimensional analysis of an iterative optimal control algorithm. Multidimensional Systems and Signal Processing 11(1-2), 109-124.

Roesser, R. P. (1975). A discrete state-space model for linear image processing. IEEE Transactions on Automatic Control, 20(1), 1-10.

Rogers, E., \& Owens, D. H. (1992). Stability analysis for linear repetitive processes, Lecture Notes in Control and Information Sciences Series, Vol. 175, Berlin: Springer Verlag.

Rogers, E., Galkowski, K., Gramacki, A., Gramacki, J., \& Owens, D. H. (2002). Stability and controllability of a class of 2D linear systems with dynamic boundary conditions. IEEE Transactions on Circuits and Systems I: Fundamental Theory and Applications 49(2), 181-195.

Sule, V. R.\& Rogers, E. A behavioral approach to the control of discrete linear repetitive processes. Systems and Control Letters 53(1), 79-88.

Valcher, M. E.(2000). Characteristic cones and stability properties of two-dimensional autonomous behaviors. IEEE Transactions on Circuits and Systems I: Fundamental Theory and Applications, 47(3), 290-302.

Wood, J. (2000). Modules and behaviours in nD systems theory. Multidimensional Systems and Signal Processing, 11, 11-48.

Wood, J., Oberst, U., Rogers, E., \& Owens, D. H. (2000). A behavioural approach to the pole structure of 1D and $\mathrm{nD}$ linear systems. SIAM Journal of Control and Optimization, 38(2), 627-661.

Zaris, P. M. (2000). Poles and zeros of nD linear systems. PhD Thesis, Department of Electronics and Computer Science, University of Southampton, UK.

Zaris, P., Wood, J., \& Rogers, E. (2001). Controllable and uncontrollable poles and zeros of nD systems. Mathematics of Control Signals and Systems, 14, 281-298.

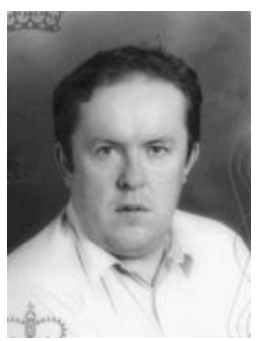

Eric Rogers was born in 1956 near Dungannon in Northern Ireland. He read Mechanical Engineering as an undergraduate in The Queen's University of Belfast UK and was awarded his PhD degree by The University of Sheffield UK for a thesis in the area of multidimensional systems theory. He has been with The University of Southampton UK since 1990 where he is currently Professor of Control Systems Theory and Design in The School of Electronics and Computer Science. Prior to moving to Southampton, he held lectureship posts in The Queen's University of Belfast UK (1984-87) and the University of Strathclyde UK (1988-90). His current major research interests include multidimensional systems theory and applications, with particular emphasis on behavioral systems theory approaches and systems with repetitive dynamics, iterative learning control, flow control, and active control of microvibrations. He is currently the editor of The International Journal of Control, an associate editor of Multidimensional Systems and Signal Processing, and joint editor of The Taylor and Francis research book series on Systems and Control. In addition, he has served extensively on IEEE, IFAC and IEE technical committees and acted as a consultant to numerous companies and government agencies in the UK and abroad. 